\title{
Effects of Exercise Training on Handgrip Strength in Older Adults: A Meta-Analytical Review
}

\author{
Berit Kristin Labott ${ }^{\mathrm{a}}$ Heidi Bucht ${ }^{\mathrm{a}}$ Mareike Morat ${ }^{\mathrm{b}}$ Tobias Morat ${ }^{\mathrm{a}}$ \\ Lars Donath $^{\mathrm{b}}$ \\ anstitute of Movement and Sport Gerontology, German Sport University, Cologne, Germany; ${ }^{\mathrm{b}}$ Institute of Exercise \\ Science and Sport Informatics, German Sport University, Cologne, Germany
}

\section{Keywords}

Aged $\cdot$ Exercise $\cdot$ Physical fitness $\cdot$ Hand strength $\cdot$ Muscle strength dynamometer

\begin{abstract}
Background: Handgrip strength measurements are feasible with older adults and a reliable indicator for vitality, physical function, and several risk factors in the ageing process. Interventions with exercise training induce a variety of strength, balance, and endurance improvements. The pooled transfer effects of exercise training on handgrip strength has not been investigated to date. Thus, the objective of this metaanalytical review is to examine the effects of different exercise training on handgrip strength in healthy communitydwelling older adults of 60 years or older. Methods: The literature search was conducted in three databases (PubMed, Web of Science, SPORTDiscus) using the following search terms with Boolean conjunctions: (hand grip* OR grip strength OR grip power) AND (sport* OR train* OR exercis* $O R$ strength $O R$ intervention $O R$ endurance $O R$ resistance $O R$ balance OR aerob*) AND (old* OR elder* OR senior*). Nonrandomized and randomized controlled trials with an exercise training and handgrip strength as the outcome param-
\end{abstract}

eter were screened. Study quality was independently assessed by two researchers using the PEDro scale. Comparison of handgrip strength between the intervention and control groups was conducted by using the hedges $g$ (including adjustment for small sample sizes), calculating standardized mean differences (SMDs). A random effects inverse-variance model was applied for statistical analysis. Results: Twentyfour trials (mean PEDro score $5.8 \pm 0.9$ ) with a total of 3,018 participants (mean age $73.3 \pm 6.0$ years) were included. Small but significant effects $(p<0.001)$ on handgrip strength were observed (SMD $0.28,95 \% \mathrm{Cl} 0.13-0.44$ ). Study heterogeneity ( $\left.{ }^{2} 56 \%\right)$ and the funnel shape for publication bias analyses were acceptable. Conclusions: Meaningful but small transfer effects of a multitude of different training approaches on handgrip strength occurred in healthy community-dwelling older adults. Handgrip strength cannot clearly be recommended to assess general functional performance for all kinds of exercise programs, whereas task-specific training and multimodal training modes seem to provide an appropriate stimulus to also improve handgrip strength.

(c) 2019 S. Karger AG, Basel

\section{KARGER}

(c) 2019 S. Karger AG, Basel

E-Mail karger@karger.com

www.karger.com/ger
Prof. Dr. Lars Donath

Department of Intervention Research in Exercise Training

Institute of Exercise Science and Sport Informatics

Am Sportpark Muengersdorf 6, DE-50933 Cologne (Germany)

E-Maill.donath@dshs-koeln.de 


\section{Introduction}

Handgrip strength testing evaluates static force that is squeezed around the dynamometer, given in kilograms, pounds, or Newtons [1]. Handgrip strength assessment serves as a feasible, quick, and reliable tool to examine vitality and physical function in the elderly population. A standardized and calibrated equipment is required, especially when different assessors [2] or different brands of dynamometers are employed [3]. The test-retest reliability is considered high: The ICC (intraclass correlation coefficient) ranges between 0.91 and 0.95 for healthy older adults [4]. According to recommendations of the American Society of Hand Therapists [5], participants should be seated, with the shoulder adducted and neutrally rotated, the elbow flexed at $90^{\circ}$, and the forearm and the wrist in a neutral position. This society recommends three trials of grip strength and suggests using the best trial for further analysis [5].

Beside other declines of physical function (endurance, strength, balance capacity), aging people show a decrease in handgrip strength [6]. Women have weaker handgrip strength compared to men [6-8]. The handgrip strength reflects a variety of physical function indices and is therefore regarded as an important indicator of health-related quality oflife of older adults [9]. Hence, handgrip strength predicts disability, morbidity, and mortality [10], accelerated dependency in activities of daily living and cognitive decline in the most elderly (over 85 years of age) [11]. A weak handgrip strength is also associated with hypertension, coronary heart disease, peripheral arterial disease, heart failure, stroke, or chronic obstructive pulmonary disease [8]. Furthermore, handgrip strength is negatively associated with physical frailty even when the effects of body mass index and arm muscle circumference are considered [12].

Strength, endurance, and balance training have been shown to attenuate numerous adverse effects of biological aging [13]. The occurrence and magnitude of potential transfer effects of these interventions with exercise training on handgrip strength as a well-established and feasible surrogate parameter in community-dwelling older adults have not yet been addressed systematically. Therefore, the objectives of the present systematic review and meta-analysis were: (1) to calculate the effects of numerous interventions with exercise training on handgrip strength in healthy community dwellers, (2) to describe training characteristics for older adults, and (3) to provide recommendations for future studies.

\section{Materials and Methods}

\section{Search Strategy and Study Selection}

The PRISMA guidelines were used for reporting this meta-analytical review [14]. A literature search was independently conducted by two researchers (B.K.L. and H.B.) in three health- and sportrelated databases (PubMed, Web of Science, SPORTDiscus). The search was conducted from November 25, 2018 until December 1, 2018. The relevant search terms were divided into three search levels and were combined with Boolean conjunctions (AND/OR). On the first search level "hand grip* OR grip strength OR grip power," on the second level "AND (sport* OR train* OR exercis* OR strength OR intervention OR endurance OR resistance OR balance OR aerob*)" and on the third level "AND (old* OR elder* OR seniors*)" were used as search terms. The screening process consisted of title, abstract, and full-text screening. First, all resulting titles of the search were screened manually by the two researchers (B.K.L. and H.B.) and discussed for eligibility. All chosen articles were then transferred to a reference management software (Citavi 6.3, Swiss Academic Software GmbH, Waedenswil, Switzerland). Both researchers screened abstracts separately and their inclusion was discussed afterwards. Consent has been found upon the independent search procedure and through discussion. Irrelevant articles were excluded and the full texts were screened for the final inclusion/ exclusion of the studies. The inclusion and exclusion criteria were defined based on the PICOS approach [population (P), intervention (I), comparators (C), main outcome (O) and study design (S)].

Only full-text articles published in English and with a target group of healthy, community-dwelling older adults of 60 years and older $(\mathrm{P})$ were included in the meta-analysis. Further inclusion criteria were that studies had to be intervention studies (I) with an exercise intervention in the form of a controlled trial (C). There had to be at least one control group per study. Handgrip strength $(\mathrm{O})$ had to be the main outcome and pre- and post-testing had to be performed (S).

The exclusion criteria were additional diet changes or nutrition supplements as part of the intervention. Furthermore, the authors aimed at investigating community-dwelling, healthy older adults, so that older adults living in a nursing home or receiving long-term care as well as older adults with mental decline, chronic diseases of the heart or vascular system, orthopaedic conditions, diabetes, sarcopenia, frailty, or obesity were excluded from the analysis.

\section{Methodological Quality}

To evaluate the methodological quality of the included studies, a scale by the Physiotherapy Evidence Database (PEDro scale) with 11 dichotomous items was used [15]. The two researchers independently rated all included studies and came to a consensus on every item after completing the individual rating (online suppl. table 1; see www.karger.com/doi/10.1159/000501203 for all online suppl. material). Researchers were not blinded to authors, place of publication, or results of the studies.

\section{Data Extraction}

Handgrip strength outcomes (in kilograms, pounds, or Newtons) were extracted and transferred to a separate excel sheet that contained the equations for all relevant calculations that are described in the statistical analysis section. Furthermore, other relevant study information (author, year, number of participants, intervention groups, and details about the intervention) were extracted and compiled in Table 1. 


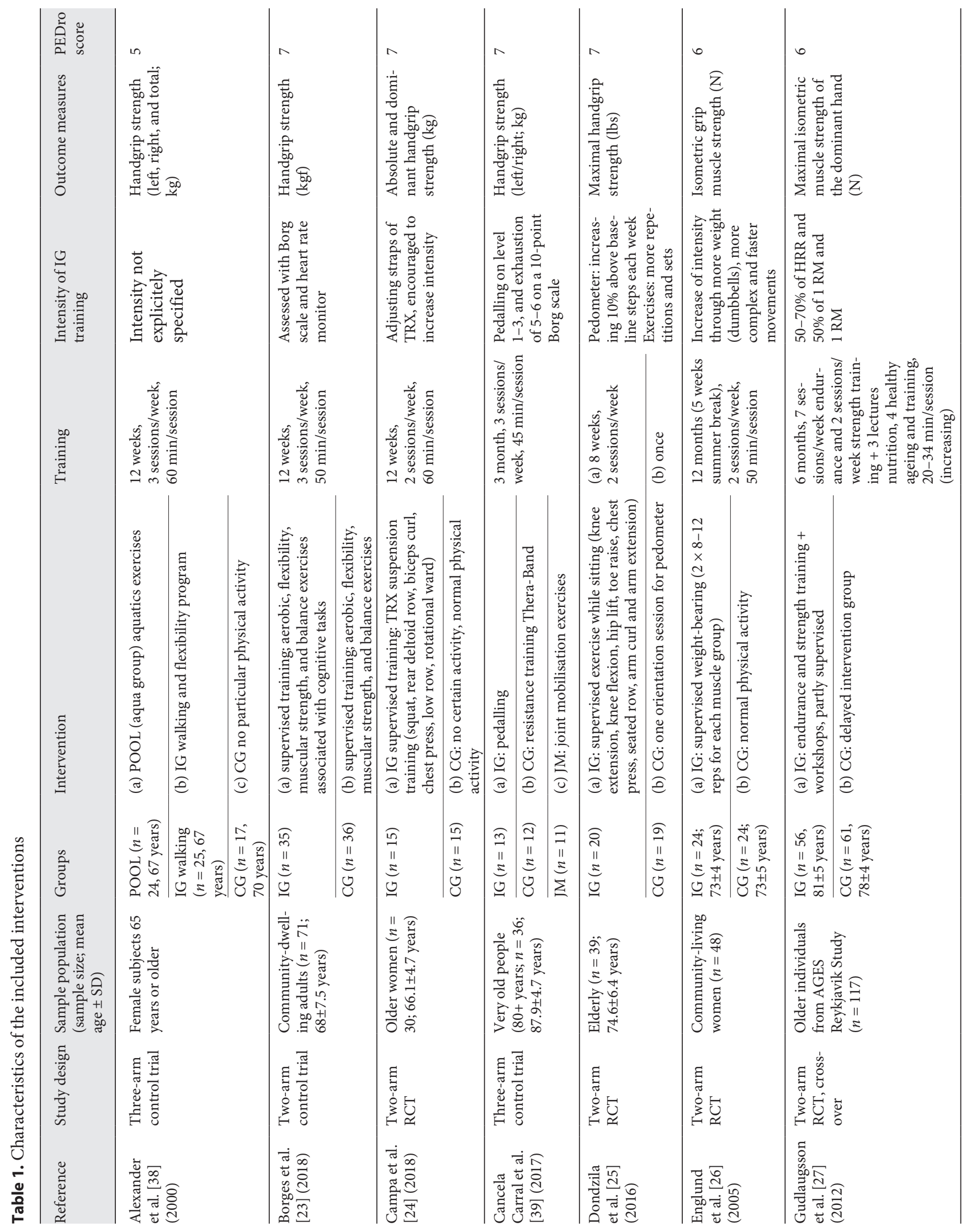




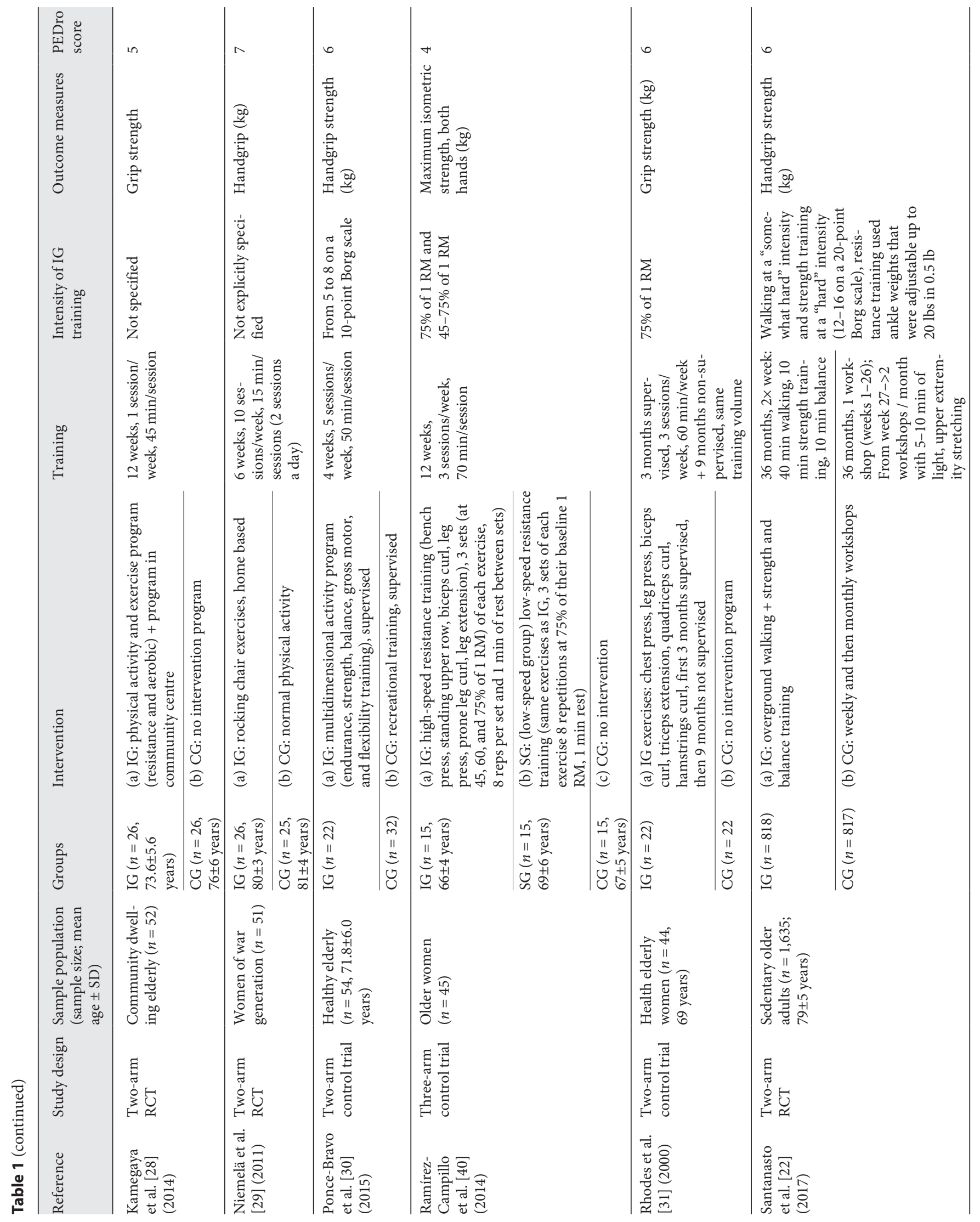




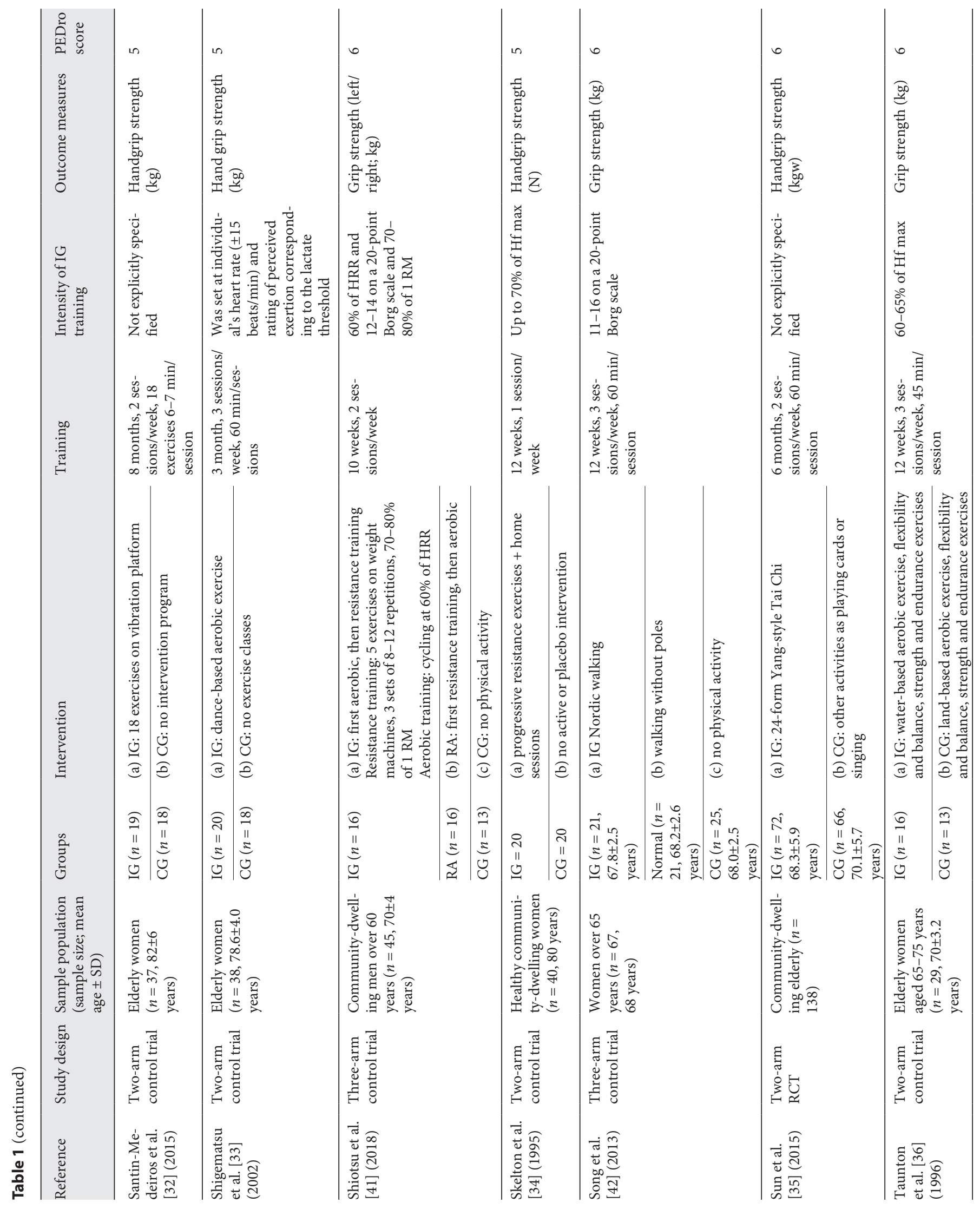




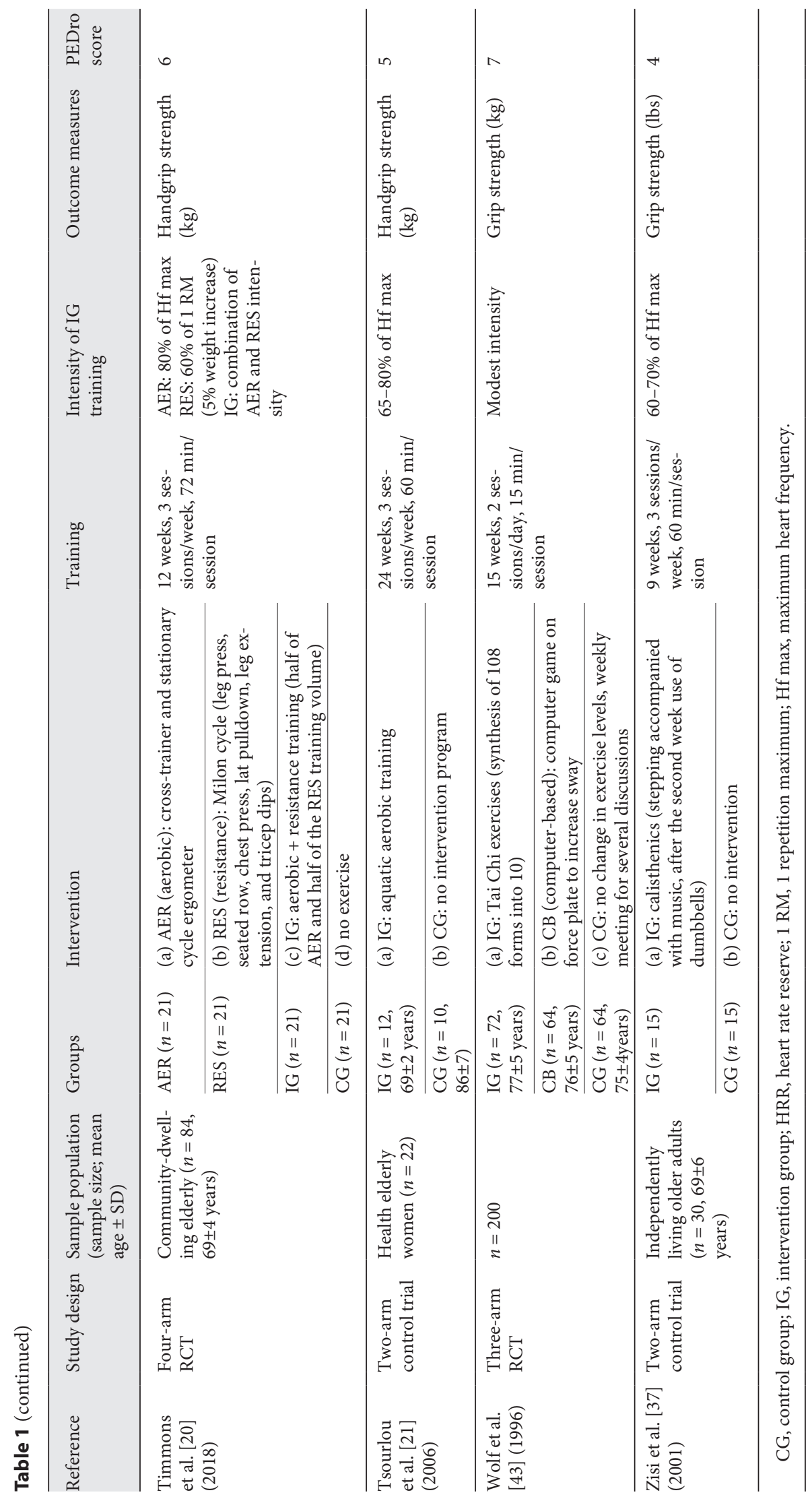




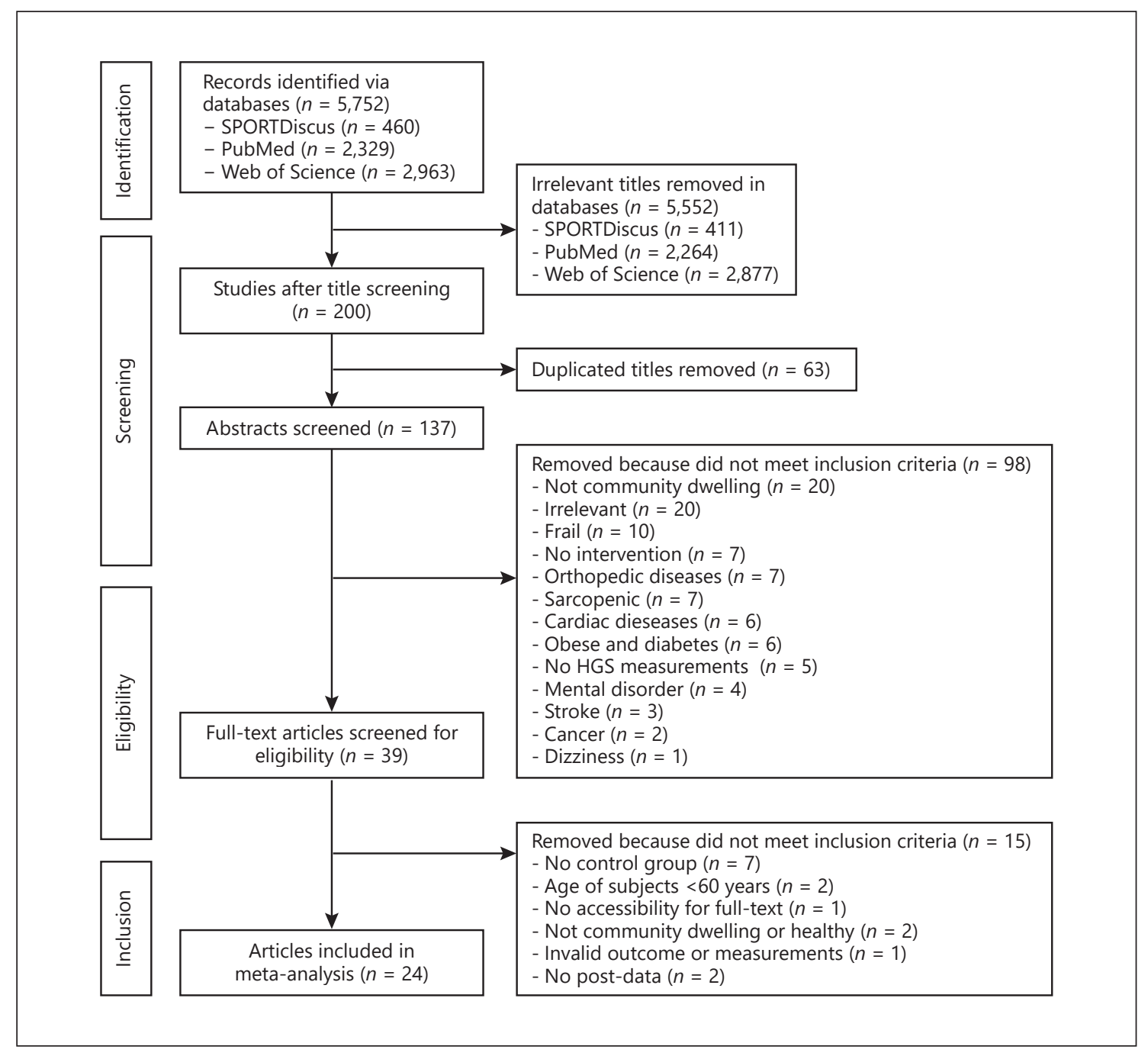

Fig. 1. Flow of study screening and selection.

\section{Statistical Analysis}

Standardized mean differences (SMD, with 95\% confidence intervals [CIs]) were calculated for each study according to Hedges' (adjusted) g, which can be used for diverged and small sample sizes [16]. Negative values (-) reflect negative effects. The Cochrane Review Manager Software (RevMan 5.3.5, Cochrane Collaboration, Oxford, UK) was used to conduct the inverse-variance method [17]. Analyses were conducted using the random effects model [18]. The forest plot was generated for the respective outcome measure. The following scale was used to evaluate the standardized mean differences (SMDs) [19]: 0-0.19 = negligible effect, 0.20$0.49=$ small effect, $0.50-0.79=$ moderate effect, and 0.80 and higher = large effect. Study heterogeneity was assessed using $I^{2}$. A funnel plot was created to examine potential publication bias by comparing the effect sizes direction with the standardized mean differences. All statistical analyses were performed within the Cochrane Review Manager Software.

\section{Results}

\section{Trial Flow}

In the three databases, 5,752 relevant articles were initially detected (Fig. 1). After removing all irrelevant studies based on the title and removing duplicates, 137 articles remained for abstract screening. Ninety-eight studies did not meet the inclusion criteria. Thirty-nine articles were left for full-text screening. Three of them were excluded during data extraction because of lacking postvalues for handgrip measurements. The authors were contacted in order to get the data for post-measurements which were not reported in the published version of the article. However, only one of the respective authors [20] reacted to the request and sent the missing post-values. 
Finally, 15 studies were excluded based on the defined exclusion criteria. For the final meta-analysis, 24 studies were included. All studies were published between 1995 and 2018.

\section{Study Population}

In the 24 trials, 3,018 healthy community-dwelling older adults with a mean age of $73.3 \pm 6.0$ years were enrolled. The mean sample size was $81 \pm 263$, with a range from 22 [21] to 1,635 [22] participants.

\section{Study Quality}

Seventeen studies used a two-armed design [21-37], six studies used a three-armed design [38-43], and one study [20] used a four-armed design. The mean study quality was $5.8( \pm 0.9)$, ranging from $4[37,40]$ to $7[23-25$, $29,39,43]$. None of the included studies blinded participants or instructors.

\section{Scope of the Studies}

Training types of all 24 studies included aquatic exercise, walking, flexibility, TRX-training, home-trainer exercise, strength training in different forms, training on a vibration platform, dance, Tai Chi, exergames balance training, calisthenics, and multi-dimensional training regimes. Intervention durations ranged from 4 weeks [30] to 36 months (mean $22 \pm 30$ weeks) [22], whereas the majority of the interventions lasted for 8-15 weeks. Session duration varied between $15[29,43]$ and 72 [20] min per session (mean $51 \pm 15$ min per session) with most of the sessions lasting $60 \mathrm{~min}$. Training frequency varied between one session per week [34] and two sessions per day $[29,43]$ (mean $3 \pm 2$ sessions per week) with mostly $2-3$ sessions per week. The training intensity was measured differently with the Borg scale, 1 repetition maximum (1 RM), or maximum heart frequency (Hf max). Using the Borg rating of perceived exertion (RPE) scale the intensity ranged from 11 to $16[22,41,42]$, and using the RPE ratio scale the intensity varied from 5 to $8[30,39]$. Five studies $[20,27,31,40,41]$ measured the training intensity with $1 \mathrm{RM}$ ranging from 45 to $80 \%$. The intensity measured with heart frequency varied from 60 to $80 \%$ of the maximum heart frequency $[20,21,27,34,36,37,41]$. Two studies did not report the intensity level $[28,35]$ and one study [23] used the Borg scale and heart rate monitor for assessing intensity but did not report values. One study reported not to have monitored training intensity [29]. Wolf et al. [43] reported modest training intensity in Tai Chi, and Campa et al. [24] encouraged the participants to increase intensity individually by changing position towards the

Effects of Training on Handgrip Strength in Older Adults anchor point of TRX. Englund et al. [26] increased the training intensity by using heavier dumbbells and by doing more complex and faster movements. Dondzila et al. [25] increased the walking intensity by $10 \%$ of baseline steps each week and the resistance training by increasing repetition and sets of exercises. Alexander et al. [38] used water exercise equipment for the aquatics exercise group to increase the intensity while the walking group exercised at a comfortable pace. Exercises increased progressively in the vibration platform training of Santin-Medeiros et al. [32]. The focus of the majority of the 24 included studies was on multimodal training, including aerobic and resistance exercises [20, 22, 23, 27, 28, 30, 36, 41].

\section{Risk of Bias}

The funnel plot (see online suppl. Fig. 1) shows a triangular funnel-shape with few outliers. The number of studies on the left and right side of the dashed SMD line seems equally distributed. Normally, studies with small sample sizes build the basis of the triangle. There is only one study [21] with a small sample size at the right bottom of the funnel plot. This two-armed controlled trial included 22 participants, 12 in the intervention group, and analysed the effects of a 24-week aquatic aerobic training. There are more studies with a bigger sample size of 30-70 participants, located in the middle of the funnel shape. The study with the biggest sample size [22] is clearly identified to the top left, outside of the funnel shape. This twoarmed controlled trial included 1,635 participants, with 818 in the intervention group, and analysed the effects of a combined walking training the intervention group performed twice a week over 36 months.

\section{Data Analysis}

Small effects were observed for handgrip strength outcome ( $p<0.001$, SMD 0.28, 95\% CI 0.13-0.44, $I^{2}=56 \%$; Fig. 2) in favour of exercise training groups compared to control groups.

\section{Discussion}

To the best of the authors' knowledge, no previous systematic review with meta-analysis has addressed potential transfer effects of interventions with exercise training on handgrip strength serving as a well-established surrogate parameter of vitality and physical function in healthy community-dwelling older adults. Measuring handgrip strength is an inexpensive, quick, and objective means to evaluate physical frailty [44] or change in performance 


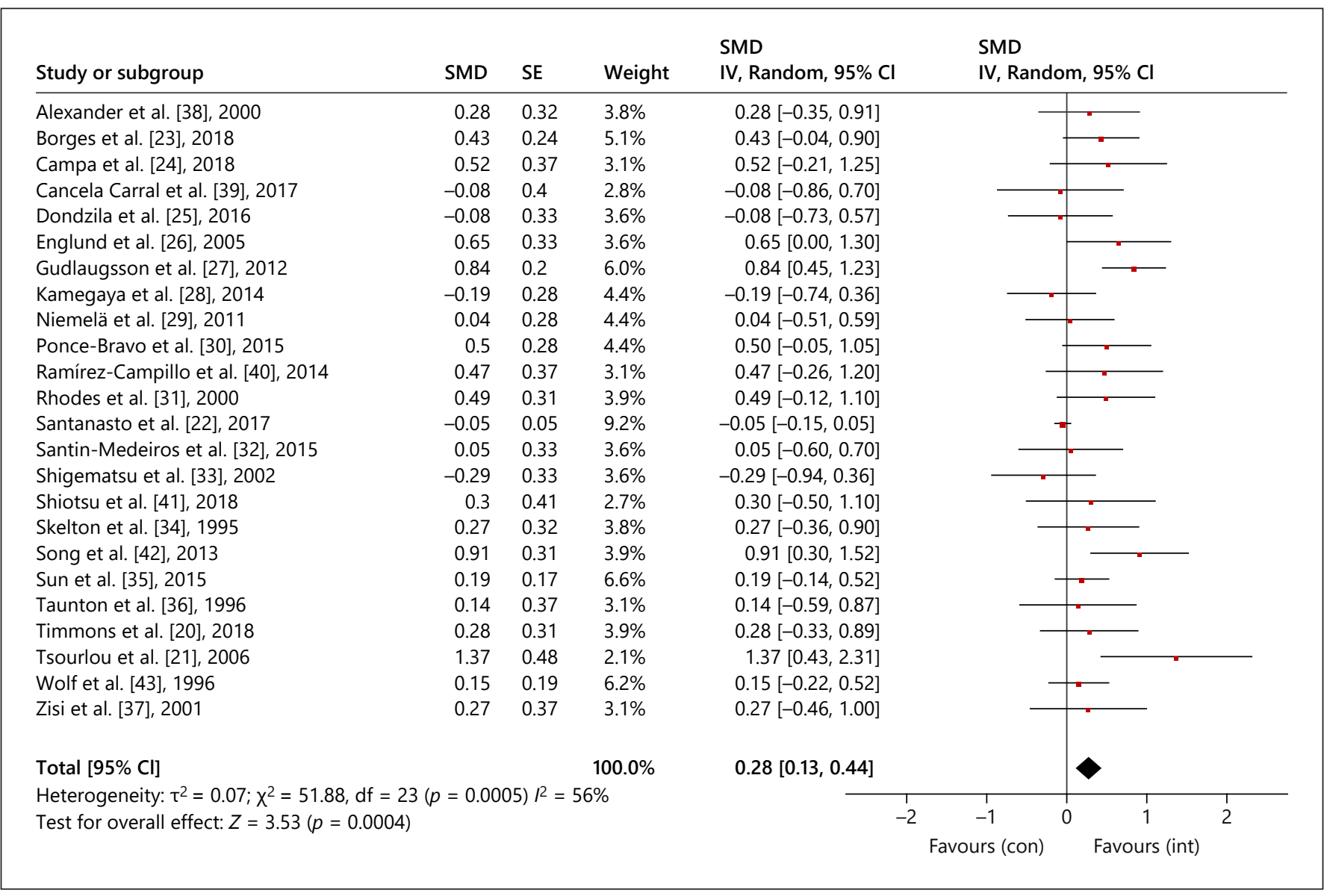

Fig. 2. Forest plot of the analysis on handgrip strength between the intervention groups (int) and the control groups (con). SE, standard error; IV, independent variable; CI, confidence interval; SMD, standardized mean difference.

[45] in small and large samples. Furthermore, handgrip strength measurements can be used to identify older adults who are sarcopenic, frail, and malnourished [46]. This meta-analytical review provides a solid estimate of exercise-induced transfer effects of various interventions with exercise training on handgrip strength. The results of this review displayed small effects of a variety of interventions on handgrip strength measures.

In order to evaluate the effects of interventions with a measurement tool, i.e. handgrip strength dynamometer, besides reliability and validity, the standard error of measurement (SEM) and minimal detectable change (MDC) have to be known. In a recent systematic review, Bohannon [46] included 17 studies about the reliability and MDC of handgrip strength measurements in different older target groups. Four of the included studies included older community-dwelling adults without any disease. The review demonstrated a relative reliability with ICC >
0.91 in these four studies with 211 participants with a mean age of 75.2 years [46]. Regarding absolute reliability, these four studies used the variables SEM, technical error of measurement (TEM) and least significant change (LSC). The SEM was 3.9 and $1.9 \mathrm{~kg}$, TEM was 1.6 (left hand) and 2.2 (right hand), and LSC was $6.0 \mathrm{~kg}$. These four relevant studies were evaluated by Bohannon [46] regarding their study quality with scores between 9 and 13 on a 14-point scale. If one takes the best results for absolute reliability for older community-dwelling older adults from Bohannon's review [46], a change (for example via an intervention) above $1.6 \mathrm{~kg}$ can be interpreted as a real change.

Measuring handgrip strength with a dynamometer can be a useful assessment tool for determining the efficacy of different treatments and for an overall fitness assessment [45]. The studies included in this meta-analysis measured handgrip strength as a general parameter of the 
state of physical function but they did not include handgrip-specific exercises. In fact, the variety in exercise mode, duration, and intensity was large. For most of the studies, the "task-specificity principle" of neuromuscular adaptation, which was manifested for balance training, seems to apply: if the positions that are tested in pre- and post-testing are not trained during the intervention, no improvement compared to the control group is revealed $[47,48]$. The outcome of this meta-analytical review supports the transfer of this idea of task specificity on handgrip strength training and measurements. Effects are more likely to appear when the tested tasks are closely related to the training contents [47-49]. Only small effects in favour of the exercise training groups, which were training with different training modes not specifically addressing handgrip strength, were found. In one of the included studies with the largest effect sizes in favour of the intervention group, participants trained with dumbbells, which included gripping of the weights [26]. Corroboratively, in another study with large effects, participants did Nordic walking [42], which included a repetitive movement similar to a gripping task by holding the pole and executing the correct Nordic walking technique. In contrast to handgrip strength assessment, Nordic walking improves the muscular endurance component and capacity instead of maximum strength, necessary for handgrip measurements. In most of the other studies, showing positive effect without task-specific training, transfer effects might account for positive changes. Additionally, it has to be taken into account that measurements of handgrip strength are usually performed isometrically, whereas exercises were mostly performed dynamically. To summarise, handgrip strength would benefit from training that mainly targets upper extremity exercises in which handgrip movements are performed.

As was mentioned, exercise mode, duration, and intensity varied among the studies included in this metaanalysis, and so did the changes in handgrip strength. As for training mode, improvements in handgrip strength indices were not only seen in interventions with pure strength/resistance training, but also in multimodal interventions as well as in a Nordic walking intervention. Interestingly, the majority of the studies that featured large effects in favour of the intervention group followed a multimodal approach to exercise training. It is assumed that handgrip strength capacity benefits more from incorporating different training focuses (strength, balance, flexibility, endurance). This finding should be taken into consideration when evaluating the efficacy of interventions with exercise training [48].

Effects of Training on Handgrip Strength in Older Adults
Almost all studies in this review met the recommendations of more than 12 weeks of intervention, except two studies that lasted less than 12 weeks $[29,30]$. No clear relationship is apparent between the duration of the intervention and the changes in handgrip strength. One study with only 6 weeks of daily rocking chair exercise found a slightly significant change in handgrip strength in favour of the intervention group [29]. Greater changes in handgrip strength were measured after a 9-week calisthenics intervention [37]. On the other hand, the study with the longest intervention duration (36 month) did not show any effects in favour of the intervention group [22]. This finding can be attributed to the control conditions that were requested to complete home exercises. Additionally, the training frequency differed a lot between the studies, so that a statement on the effect of the study duration is hardly possible. A further factor potentially influencing effects on handgrip strength might be training intensity. When the intensity of the intervention was reported as between 60 and $80 \%$ of $1 \mathrm{RM}$, handgrip strength increased. Of those measuring intensity with $1 \mathrm{RM}$ as reference, the intervention of Gudlaugsson et al. [27], where the participants trained with $6 \mathrm{RM}$, had the greatest changes in favour of the intervention group. In the studies using the Borg scale as the reference, handgrip strength increased when the perceived exertion was reported as "somewhat hard" or "hard" (12-16) [41, 42] with the exception of the study by Santanasto et al. [22]. Regarding Hf max, interventions with a variation of 15$20 \%$ between minimum and maximum heart frequency in training seem to be the most effective [21, 27]. Continuous training with $80 \% \mathrm{Hf} \max$ [20] is not more effective than training with $60 \% \mathrm{Hf} \max$ [41].

Due to the high variability of the training designs of all studies, it is not possible to tell the influence of specific training modalities (training mode, duration, or intensity) on changes in handgrip strength. The confidence intervals of four studies that did not touch the zero-effect line indicate a large effect in favour of the intervention groups $[21,26,27,42]$. These studies included weightbearing training over 12 months [26], endurance training combined with strength training over 6 months [27], aquatic aerobic training over 3 months [21], and Nordic walking over 3 months [42], and therefore obviously differed in terms of exercise mode, duration, and intensity despite comparable effects. Only five of the included studies trended towards a favourable effect for the control group [22, 25, 28, 33, 39]. These studies consisted of seated strength training [25], resistance and aerobic exercises [28] and dance-based aerobic exercises [33]. Additional- 
ly, the participants of one study did either low-loaded pedalling or unspecific exercises using elastic bands [39], and the remaining comprised workshops, strength, or balance training [22]. Again, no prototype for mode, duration, or intensity of the training can be found. The reasons for unchanged handgrip strength might be attributed to the age of the participants [39], a short intervention period [25], the lack of weekly training sessions [28], or short duration of single training sessions $[22,33]$.

Older adults who do not train beside the normal activities of daily living lose their body strength and arm strength [50]. Therefore, based on the present meta-analytical review, the authors recommend exercise training for community-dwelling older adults in order to improve or maintain functions of activities of daily living and to remain independent. The overall effect of the studies analysed in this meta-analysis and the overlapping intervals indicate that there is neither a clear effect in favour of the control nor the intervention group after an unspecific training intervention. Thus, if the aim is to increase handgrip strength, the authors recommend at least 9 weeks of task-specific training with variable heart frequency, $75 \%$ $1 \mathrm{RM}$, and perceived "somewhat hard" or "hard" exertion. Whether the same is true for frail older adults or for older adults living in care facilities remains unclear and needs to be addressed in future analyses. Illness and frailty could have a strong impact on the results so that these target groups need to be investigated separately. This meta-analysis therefore focused on the effects of training on handgrip strength of a healthy population only. According to the results, handgrip strength cannot clearly be recommended as an alternative outcome to assess general functional performance for all kinds of exercise programs. It can be assumed though that handgrip strength changes after multimodal interventions and thus can be used as an outcome measure for this training modality. However, it is questionable whether pure lower extremity exercise or endurance exercise programs, for example, substantially alter handgrip performance, even if they cause other performance changes.

\section{Strengths and Limitations}

This meta-analytical review was performed in accordance with the PRISMA statement [14]. Despite notable but acceptable heterogeneity of the included studies (intervention programmes, number of study arms, sample sizes), our findings provide a structured view on the transfer effects of interventions with exercise training on handgrip strength, independent of the underlying exercise regimen. It needs to be kept in mind that although handgrip strength is a feasible and easily applicable tool of vitality and functioning assessment in older adults, few interventions with exercise training include this outcome even if muscle strength has been targeted. In most of the included studies the training procedure and performed exercises were clearly described. The different interventions might bias the effects as none primarily promoted handgrip strength explicitly. The relative reliability of handgrip strength measurements is very good; however, the measurement tool showed weakness in absolute reliability. Furthermore, the achieved intervention effects have to be interpreted allowing for SEM and MDC.

\section{Conclusion}

The present meta-analysis focused on healthy, noninstitutionalized older adults and revealed evidence that several interventions provide an appropriate training for improving handgrip strength, especially those with multimodal training. Small transfer effects can be explained due to the lack of task-specific exercises, the lack of weekly training sessions, or short duration of single training sessions. For meaningful conclusions, substantial changes in handgrip strength may be required to confidently conclude that real changes have occurred over time. Still, the effectiveness of exercise training is not to be underestimated. Beyond that, handgrip strength cannot clearly be recommended as an alternative outcome to assess general functional performance for all kinds of exercise programs, possibly apart from multimodal training modes. Furthermore, the effects of interventions with exercise training on handgrip strength in specific target groups, such as institutionalized or frail older adults, must be investigated in the future.

\section{Acknowledgements}

This meta-analytical review was prepared within the module "SBG11 - Review" in the master study program "sport and movement gerontology" (Master of Science) at the German Sport University Cologne.

\section{Statement of Ethics}

The authors have no ethical conflicts to disclose.

\section{Disclosure Statement}

The authors have no conflicts of interest to declare. 


\section{References}

1 Massy-Westropp NM, Gill TK, Taylor AW, Bohannon RW, Hill CL. Hand Grip Strength: age and gender stratified normative data in a population-based study. BMC Res Notes. 2011 Apr;4(1):127.

2 Mathiowetz V, Kashman N, Volland G, Weber K, Dowe M, Rogers S. Grip and pinch strength: normative data for adults. Arch Phys Med Rehabil. 1985 Feb;66(2):69-74.

3 Smidt N, van der Windt DA, Assendelft WJ, Mourits AJ, Devillé WL, de Winter AF, et al. Interobserver reproducibility of the assessment of severity of complaints, grip strength, and pressure pain threshold in patients with lateral epicondylitis. Arch Phys Med Rehabil. 2002 Aug;83(8):1145-50.

4 Bohannon RW, Schaubert KL. Test-retest reliability of grip-strength measures obtained over a 12-week interval from communitydwelling elders. J Hand Ther. 2005 Oct-Dec; 18(4):426-7.

5 American Society of Hand Therapists. Clinical assessment recommendations. 1st ed. Mount Laurel: ASHT; 1981.

6 Dodds RM, Syddall HE, Cooper R, Kuh D, Cooper C, Sayer AA. Global variation in grip strength: a systematic review and meta-analysis of normative data. Age Ageing. 2016 Mar 45(2):209-16

7 Kuh D, Bassey EJ, Butterworth S, Hardy R, Wadsworth ME; Musculoskeletal Study Team. Grip strength, postural control, and functional leg power in a representative cohort of British men and women: associations with physical activity, health status, and socioeconomic conditions. J Gerontol A Biol Sci Med Sci. 2005 Feb;60(2):224-31

8 Leong DP, Teo KK, Rangarajan S, Lopez-Jaramillo P, Avezum A Jr, Orlandini A, et al.; Prospective Urban Rural Epidemiology (PURE) Study investigators. Prognostic value of grip strength: findings from the Prospective Urban Rural Epidemiology (PURE) study. Lancet. 2015 Jul;386(9990):266-73.

9 Musalek C, Kirchengast S. Grip strength as an indicator of health-related quality of life in old age - a pilot study. Int J Environ Res Public Health. 2017 Nov;14(12):E1447.

10 Sayer AA, Kirkwood TB. Grip strength and mortality: a biomarker of ageing? Lancet. 2015 Jul;386(9990):226-7.

11 Taekema DG, Gussekloo J, Maier AB, Westendorp RG, de Craen AJ. Handgrip strength as a predictor of functional, psychological and social health. A prospective population-based study among the oldest old. Age Ageing. 2010 May;39(3):331-7.

12 Syddall H, Cooper C, Martin F, Briggs R, Aihie Sayer A. Is grip strength a useful single marker of frailty? Age Ageing. 2003 Nov; 32(6):650-6.
13 Milanović Z, Pantelić S, Trajković N, Sporiš G, Kostić R, James N. Age-related decrease in physical activity and functional fitness among elderly men and women. Clin Interv Aging. 2013;8:549-56.

14 Hutton B, Salanti G, Caldwell DM, Chaimani A, Schmid CH, Cameron C, et al. The PRISMA extension statement for reporting of systematic reviews incorporating network metaanalyses of health care interventions: checklist and explanations. Ann Intern Med. 2015 Jun;162(11):777-84.

15 Maher CG, Sherrington C, Herbert RD, Moseley AM, Elkins M. Reliability of the PEDro scale for rating quality of randomized controlled trials. Phys Ther. 2003 Aug;83(8): 713-21.

16 Ellis PD. The essential guide to effect sizes: Statistical power, meta-analysis, and the in terpretation of research results. Cambridge: Cambridge University Press; 2010. https:// doi.org/10.1017/CBO9780511761676.

17 Deeks JJ, Higgins JP. Statistical algorithms in Review Manager 5. London: The Cochrane Collaboration; 2016.

18 Borenstein M, Hedges LV, Higgins JP, Rothstein HR. A basic introduction to fixed-effect and random-effects models for meta-analysis. Res Synth Methods. 2010 Apr;1(2):97-111.

19 Cohen J. Statistical power analysis for the behavioral sciences. 2. Hillsdale (NJ): Erlbaum; 1988.

20 Timmons JF, Minnock D, Hone M, Cogan KE, Murphy JC, Egan B. Comparison of timematched aerobic, resistance, or concurrent exercise training in older adults. Scand J Med Sci Sports. 2018 Nov;28(11):2272-83.

21 Tsourlou T, Benik A, Dipla K, Zafeiridis A, Kellis S. The effects of a twenty-four-week aquatic training program on muscular strength performance in healthy elderly women. J Strength Cond Res. 2006 Nov;20(4): 811-8.

22 Santanasto AJ, Glynn NW, Lovato LC, Blair SN, Fielding RA, Gill TM, et al.; LIFE Study Group. Effect of physical activity versus health education on physical function, grip strength and mobility. J Am Geriatr Soc. 2017 Jul;65(7):1427-33.

23 Borges ML, Hotta AJ, de Souza B, Stephanie $\mathrm{M}$, et al. Impact of a dual task intervention on physical performance of older adults who practice physical exercise. Braz J Kinathrop Hum Perform. 2018;20(1):10-9.

24 Campa F, Silva AM, Toselli S. Changes in Phase Angle and Handgrip Strength Induced by Suspension Training in Older Women. Int J Sports Med. 2018 Jun;39(6):442-9.

25 Dondzila CJ, Swartz AM, Keenan KG, Harley AE, Azen R, Strath SJ. Translating exercise interventions to an in-home setting for seniors: preliminary impact on physical activity and function. Aging Clin Exp Res. 2016 Dec;28(6): 1227-35.
26 Englund U, Littbrand H, Sondell A, Pettersson U, Bucht G. A 1-year combined weightbearing training program is beneficial for bone mineral density and neuromuscular function in older women. Osteoporos Int. 2005 Sep;16(9):1117-23.

27 Gudlaugsson J, Gudnason V, Aspelund T, Siggeirsdottir K, Olafsdottir AS, Jonsson PV, et al. Effects of a 6-month multimodal training intervention on retention of functional fitness in older adults: a randomized-controlled cross-over design. Int J Behav Nutr Phys Act. 2012 Sep;9(1):107.

28 Kamegaya T, Araki Y, Kigure H, Yamaguchi $\mathrm{H}$; Long-Term-Care Prevention Team of Maebashi City. Twelve-week physical and leisure activity programme improved cognitive function in community-dwelling elderly subjects: a randomized controlled trial. Psychogeriatrics. 2014 Mar;14(1):47-54.

29 Niemelä K, Väänänen I, Leinonen R, Laukkanen P. Benefits of home-based rockingchair exercise for physical performance in community-dwelling elderly women: a randomized controlled trial. Aging Clin Exp Res. 2011 Aug;23(4):279-87.

30 Ponce-Bravo H, Ponce C, Feriche B, Padial P. Influence of Two Different Exercise Programs on Physical Fitness and Cognitive Performance in Active Older Adults: Functional Resistance-Band Exercises vs. Recreational Oriented Exercises. J Sports Sci Med. 2015 Nov; 14(4):716-22.

31 Rhodes EC, Martin AD, Taunton JE, Donnelly M, Warren J, Elliot J. Effects of one year of resistance training on the relation between muscular strength and bone density in elderly women. Br J Sports Med. 2000 Feb;34(1):1822.

32 Santin-Medeiros F, Rey-López JP, Santos-Lozano A, Cristi-Montero CS, Garatachea Vallejo N. Effects of eight months of wholebody vibration training on the muscle mass and functional capacity of elderly women. J Strength Cond Res. 2015 Jul;29(7):1863-9.

33 Shigematsu R, Chang M, Yabushita N, Sakai T, Nakagaichi M, Nho H, et al. Dance-based aerobic exercise may improve indices of falling risk in older women. Age Ageing. 2002 Jul; 31(4):261-6.

34 Skelton DA, Young A, Greig CA, Malbut KE. Effects of resistance training on strength, power, and selected functional abilities of women aged 75 and older. J Am Geriatr Soc. 1995 Oct;43(10):1081-7.

35 Sun J, Kanagawa K, Sasaki J, Ooki S, Xu H, Wang L. Tai chi improves cognitive and physical function in the elderly: a randomized controlled trial. J Phys Ther Sci. 2015 May;27(5): 1467-71. 
36 Taunton JE, Rhodes EC, Wolski LA, Donelly M, Warren J, Elliot J, et al. Effect of land-based and water-based fitness programs on the cardiovascular fitness, strength and flexibility of women aged 65-75 years. Gerontology. 1996; 42(4):204-10

37 Zisi V, Michalopoulou M, Tzetzis G, Kioumourtzoglou E. Effects of a short-term exercise program on motor function and whole body reaction time in the elderly. J Hum Mov Stud. 2001;40(2):145-60.

38 Alexander MJ, Nickel R, Boreskie SL, Searle M. Comparison of the effects of two types of fitness/flexibility programs on gait, mobility and self-esteem of older females. J Hum Mov Stud. 2000;38(5):235-68.

39 Cancela Carral JM, Pallin E, Orbegozo A, Ayán Pérez C. Effects of three different chairbased exercise programs on people older than 80 years. Rejuvenation Res. 2017 Oct;20(5): $411-9$.
40 Ramírez-Campillo R, Castillo A, de la Fuente CI, Campos-Jara C, Andrade DC, Álvarez C, et al. High-speed resistance training is more effective than low-speed resistance training to increase functional capacity and muscle performance in older women. Exp Gerontol. 2014 Oct; 58:51-7.

41 Shiotsu Y, Watanabe Y, Tujii S, Yanagita M. Effect of exercise order of combined aerobic and resistance training on arterial stiffness in older men. Exp Gerontol. 2018 Oct;111:2734.

42 Song MS, Yoo YK, Choi CH, Kim NC. Effects of nordic walking on body composition, muscle strength, and lipid profile in elderly women. Asian Nurs Res (Korean Soc Nurs Sci). 2013 Mar;7(1):1-7.

43 Wolf SL, Barnhart HX, Kutner NG, McNeely $\mathrm{E}$, Coogler $\mathrm{C}, \mathrm{Xu} \mathrm{T}$. Reducing frailty and falls in older persons: an investigation of Tai Chi and computerized balance training. Atlanta FICSIT Group. Frailty and Injuries: Cooperative Studies of Intervention Techniques. J Am Geriatr Soc. 1996 May;44(5):489-97.

44 Bohannon RW. Dynamometer measurements of hand-grip strength predict multiple outcomes. Percept Mot Skills. 2001 Oct;93(2): 323-8.
45 Innes E. Handgrip strength testing: A review of the literature. Aust Occup Ther J. 1999; 46(3):120-40.

46 Bohannon RW. Test-retest reliability of measurements of hand-grip strength obtained by dynamometry from older adults: a systematic review of research in the PubMed database. J Frailty Aging. 2017;6(2):83-7.

47 Giboin LS, Gruber M, Kramer A. Task-specificity of balance training. Hum Mov Sci. 2015 Dec;44:22-31.

48 Kümmel J, Kramer A, Giboin LS, Gruber M. Specificity of balance training in healthy individuals: a systematic review and meta-analysis. Sports Med. 2016 Sep;46(9):1261-71.

49 Donath L, Roth R, Zahner L, Faude O. Slackline training and neuromuscular performance in seniors: A randomized controlled trial. Scand J Med Sci Sports. 2016 Mar;26(3): 275-83.

50 Mayer F, Scharhag-Rosenberger F, Carlsohn A, Cassel M, Müller S, Scharhag J. The intensity and effects of strength training in the elderly. Dtsch Arztebl Int. 2011 May;108(21): $359-64$ 